\title{
CORRELACIONANDO TIPOS DE CULTURA ORGANIZACIONAL COM ESTRATÉGIAS DE REMUNERAÇÃO UTILIZANDO A TIPOLOGIA DE CHARLES HANDY
}

\author{
Giuseppe Maria Russo \\ giuseppe.russo@globo.com \\ Pontifícia Universidade Católica do Rio de Janeiro - Rio de Janeiro, RJ/Brasil \\ Patricia Amelia Tomei \\ patomei@iag.puc-rio.br \\ Pontifícia Universidade Católica do Rio de Janeiro - Rio de Janeiro, RJ/Brasil \\ Antonio José Braga Linhares \\ linhares@openlink.com.br \\ Pontifícia Universidade Católica do Rio de Janeiro - Rio de Janeiro, RJ/Brasil \\ Andre Moreira Santos \\ andresantos@pbsbrasil.com.br \\ Pontifícia Universidade Católica do Rio de Janeiro - Rio de Janeiro, RJ/Brasil
}

Recebido em 13/10/2011

Aprovado em 27/09/2012

Disponibilizado em 01/12/2012

Avaliado pelo sistema double blind review

Revista Eletrônica de Administração

Editor: Luís Felipe Nascimento

ISSN 1413-2311 (versão on-line)

Editada pela Escola de Administração da Universidade Federal do Rio Grande do Sul.

Periodicidade: Quadrimestral

Sistema requerido: Adobe Acrobat Reader.

\section{RESUMO}

Este estudo identificou as correlações entre as estratégias de remuneração e a tipologia de cultura organizacional proposta por Handy (2003) baseada nas dimensões do grau de formalização e centralização que identifica a cultura: Zeus (poder), Apolo (papéis), Atena (tarefa), e Dionísio (pessoa).

A pesquisa foi aplicada a gestores de remuneração de 76 empresas associadas ao Grupo de Permuta de Informações Salariais (Grupisa), e os questionários com um construto composto por doze componentes da remuneração organizacional foram analisados utilizando Correlação de Pearson e Regressão Múltipla.

Concluímos que as variáveis de remuneração se correlacionam de forma diferente com cada um dos tipos de cultura: (i) organizações "Zeus" devem enfatizar os fatores comportamentais em todas as esferas do sistema de remuneração; (ii) para as organizações "Apolo" a ênfase deve ser aplicada nas oportunidades de crescimento e desenvolvimento, (iii) nas organizações 
Correlacionando tipos de cultura organizacional com estratégias de remuneração utilizando a tipologia de Charles Handy

"Atena" o foco deve ser nas recompensas financeiras, e, (iv) organizações "Dionísio" devem dar ênfase à qualidade da remuneração.

Palavras-chave: estratégias de remuneração, cultura organizacional, tipologia cultural, alinhamento cultural.

\title{
CORRELATION BETWEEN ORGANIZATIONAL CULTURE AND COMPENSATION STRATEGIES USING CHARLES HANDY'S TYPOLOGY
}

\begin{abstract}
This study identified the correlations between compensation strategies and the organizational culture typology proposed by Handy (2003), based on the degree of formalization and centralization that identifies the culture: Zeus (power), Apollo (roles), Athena (task) and Dionysus (person).

A survey was performed of compensation managers at 76 companies associated with the Salary Information Exchange Group (GRUPISA), and the questionnaires with a construct composed of twelve organizational compensation components were analyzed using Pearson's correlation coefficient and multiple regression

We concluded that the compensation variables were correlated in a different fashion with each of the culture types: (i) "Zeus" organizations should emphasize behavioral factors in all spheres of the compensation system; (ii) in the case of "Apollo" organizations the emphasis should be on growth and development opportunities; (iii) in "Athena" organizations the focus should be on financial rewards, and, "Dionysus" organizations should place emphasis on the quality of compensation
\end{abstract}

Keywords: compensation strategy, organizational culture, cultural typology, cultural fit.

\section{INTRODUÇÃO}

Para o alcance dos desafios relativos à retenção e ao comprometimento dos profissionais é necessária a observância pelas áreas de Recursos Humanos do alinhamento de suas estratégias, políticas e práticas gerenciais com a cultura das organizações (Bourantas \& Papadakis, 1997; Gainey, Kelley, \& Hill, 1999; Moreira, 2008; Naves \& Coletas, 2003).

Neste contexto, percebemos que o sistema de remuneração, dentre os outros subsistemas de Recursos Humanos, é uma estratégia relevante para o alinhamento, na medida em que especifica:

o que o empregador quer de seus empregados e o que ele deve ser motivado a oferecer, (...) reconhecendo a contribuição do profissional a partir da análise de uma série de dimensões e motivações que o impele a esforçar-se mentalmente e fisicamente 
Giuseppe Maria Russo, Patricia Amelia Tomei, Antonio José Braga Linhares \& Andre Moreira Santos

e a alocar seus esforços de uma maneira que sirva aos interesses da organização. (Milgrom e Roberts, 1992, p. 92).

Estudos têm sido realizados buscando correlacionar a remuneração (compensação e recompensas) ao desempenho, mas conforme podemos observar, a partir do levantamento exploratório do tema remuneração em revistas científicas brasileiras, tem sido pouco discutido na literatura acadêmica.

O conhecimento da cultura organizacional é fundamental para a administração e implantação de estratégias e mudanças que visem melhorar o desempenho de uma organização (Russo, 2010). Entender quais são as características culturais importantes para alavancar o desempenho organizacional é extremamente relevante, principalmente com a necessidade de ser eficiente dentro de mercados cada vez mais competitivos e dinâmicos (Russo, 2010).

Como apresenta O’Neal (1992), um sistema de administração da remuneração que indique o que é esperado das pessoas pela empresa é fundamental para o sucesso empresarial. Existindo alinhamento com a estratégia da empresa, a remuneração atua no incentivo à harmonia de interesses e desenvolvimento de visões comuns dentro da realidade da cultura de determinada organização.

Com base nestes pressupostos, neste trabalho buscamos verificar se existe correlação entre tipos de cultura e os componentes estratégicos de remuneração, a partir de uma análise do impacto das estratégias de remuneração na cultura organizacional.

Este estudo é um subsídio para reforçar a importância da definição da estratégia de remuneração com base na cultura organizacional, buscando identificar, correlacionando, a estratégia de remuneração mais adequada para cada tipo de cultura organizacional.

\section{REFERENCIAL TEÓRICO}

\section{Cultura organizacional}

O conceito de fragmentação decorrente do racionalismo científico (Bateman \& Snell, 1998), permite à cultura organizacional ser estudada cientificamente, como um dos componentes que constituem as organizações. Neste sentido, a cultura organizacional resguardada a sua 
Correlacionando tipos de cultura organizacional com estratégias de remuneração utilizando a tipologia de Charles Handy

subjetividade (Srour, 1998), é vista como algo definível e suas estratégias podem ser direcionadas para atividades relacionadas à estrutura, aos procedimentos, às punições e recompensas (Bateman \& Snell, 1998).

Na história do conceito de cultura organizacional identificam-se três momentos distintos (Barbosa, 2002). O primeiro ocorreu na década de sessenta, quando o conceito de cultura organizacional era correlacionado com o movimento de desenvolvimento organizacional e com a concepção humanística dos valores organizacionais. Naquela época, a cultura organizacional era percebida como um instrumento de melhoria das organizações, mas existia baixo interesse em tratá-la como uma vantagem competitiva.

O segundo teve seu auge na década de oitenta, quando os estudos das empresas japonesas demonstraram a relevância da cultura organizacional no espaço econômico e empresarial. Naquele período, ocorreram discussões epistemológicas sobre o que é cultura organizacional, numa dimensão pragmático-substantiva, na tentativa de transformar o conceito de cultura organizacional em uma variável da estratégia gerencial e de competitividade. Como afirmam Bourantas, Anagnostelis, Mantes e Kefalas (1990), nos anos 80 surgiram novos modelos na teoria organizacional e nos desenhos de estratégia. Os pesquisadores começaram a investigar valores, credos, rituais, costumes e outras variáveis das organizações que pareciam influenciar o desempenho organizacional. Dentre as principais variáveis encontradas nos estudos, existiam as que constituíam aspectos da tradição das organizações, que passaram a ser denominadas como cultura organizacional.

O terceiro período teve início em meados da década de noventa, quando a cultura organizacional passou a ser compreendida e estudada como um ativo intangível das organizações. Naquele contexto, a cultura organizacional tornou-se uma variável mais de estratégia do que gerencial, tendo a liderança da organização um papel central na sua definição, manutenção e internalização (Schein, 1992).

Paralelamente ao desenvolvimento dos conceitos de cultura organizacional, surgiram diversos instrumentos objetivando identificá-la de forma científica. Segundo Igo e Skitmore (2006) existem diversos instrumentos e métodos que podem ser usados para caracterizar a cultura organizacional. Os principais, citados pelos autores, são o Organizational Culture Profile de O'Reilly, Chatman e Caldwell (1991) e o Culture Assessment Instrument de Cameron e Quinn 
Giuseppe Maria Russo, Patricia Amelia Tomei, Antonio José Braga Linhares \& Andre Moreira Santos

(1999). Além desses, muitos outros foram apontados por Tomei, Russo e Antonaccio (2008), entre eles: as tipologias de Handy (1980), Daft (2000) e Trompenaars (1994).

Os modelos ou tipologias culturais não correspondem à realidade organizacional como um todo, mas são construções teóricas que permitem que se busque a generalização de um objeto, quando definida certa visão ou abordagem, funcionando como recurso metodológico, como indica Freitas (1991). Entretanto, quando há necessidade de lidar com múltiplas dimensões, as tipologias são utilizadas como uma alternativa para prover a simplificação dos significados para acessar a cultura organizacional (Ankrah e Langford, 2005). Dessa forma, as tipologias ajudam a compreender a cultura organizacional.

Diversos autores (Baldacchino, 1997; Bourantas et al., 1990; Gainey, Kelley, \& Hill, 1999; Gomide \& Martins, 1997, Naves \& Coleta, 2003) utilizaram a tipologia de Charles Handy em seus estudos, demonstrando a sua importância para o entendimento da cultura organizacional.

Handy (1980) discorreu sobre a diversidade das estruturas organizacionais e dos sistemas administrativos, assim como, levantou questionamento sobre a diversidade da cultura organizacional, tendo em vista que ela é influenciada por diversos fatores e se reflete nas estruturas e nos diversos sistemas organizacionais, como o sistema de remuneração. Aliado ao fato de que a tipologia de Handy (1980) foi validada por Bourantas et al. (1990), assim como, por Gomide e Martins (1997) em estudos empíricos com grandes amostras, este estudo utilizou a tipologia de Handy (1980) para investigar a existência de correlação entre a cultura e as estratégias de remuneração organizacional.

Handy (1980) utilizou-se de partes dos trabalhos de Roger Harrison que classifica as culturas em burocrática, de tarefa, de poder e democrática, falando sobre a ideologia e o caráter organizacionais. Além disso, elaborou uma tipologia cultural que considera como os canais de poder são refletidos nas organizações em determinadas categorias culturais. Como indica Harrison (1972), a ideologia organizacional afeta o comportamento dos empregados e suas interações com o ambiente.

Handy (1980) fez uma associação destas categorias com os deuses gregos para indicar que a administração não é uma ciência exata, mas um processo criativo e político que deve muito à cultura e à tradição do lugar e do momento. Porém, existem certos padrões no comportamento 
Correlacionando tipos de cultura organizacional com estratégias de remuneração utilizando a tipologia de Charles Handy

das pessoas que servem de apoio na decisão dos administradores e cuja identificação é possível. O uso da mitologia por Handy (1980), representada pelos deuses gregos Zeus, Apolo, Atena e Dionísio, visa a identificar esses padrões e relacionar sua representatividade cultural com a filosofia administrativa mais aderente. Como indica Bourantas et al., (1990), esses deuses foram escolhidos para representar os quatro principais pilares da sabedoria e descrever a cultura organizacional. É importante destacar que o autor indica que cada cultura não é encontrada de forma única e não deve ser classificada como ruim ou boa, e sim adequada ou inadequada para o profissional ou a empresa.

O primeiro tipo chamado de Cultura do Poder ou Cultura-de-Clube é representada pelo deus do Olimpo, soberano da Acrópole, chamado Zeus, representando o poder patriarcal, benevolente, impulsivo, carismático e irracional. A Cultura do Poder é representada por uma estrutura em forma de teia, onde o poder encontra-se no núcleo central. Esse tipo de cultura apresenta poucos procedimentos e regras, sendo que as decisões são tomadas com base no equilíbrio entre a influência e as razões lógicas. As organizações com este tipo de cultura normalmente são estruturadas por função ou linha de produtos. É um tipo de cultura que valoriza os funcionários que estão em volta da teia de influência de Zeus. Para manter seus súditos, Zeus dá recompensas e remuneração de acordo com o relacionamento, e não em função da estrutura formal (Bourantas et al., 1990).

O segundo tipo chamado de Cultura dos Papéis é representada pelo deus Apolo, guerreiro sagaz, deus da ordem e das regras. A Cultura dos Papéis é representada na figura do templo grego, pela sua beleza e força de seus pilares. As funções e especialidades são os pontos significativos dos pilares desse tipo de cultura, onde a interação entre a alta administração e a base é controlada por procedimentos para cada papel (descrições e definições), com regras para a solução dos conflitos. O ambiente para os funcionários das organizações com esse tipo de cultura é muito seguro e previsível, onde as atividades são fixas e há divisão funcional do trabalho. Os funcionários alinhados com a cultura Apolo mantêm-se, de forma disciplinada, no aguardo das oportunidades que possam surgir na empresa. As pessoas são parte de uma engrenagem na estrutura organizacional (Bourantas \& Papadakis, 1997).

O terceiro tipo chamado de Cultura de Tarefa é representado pela deusa Atena, que possui a missão de ajudar seus súditos, com orientação para a vitória. Esse tipo de cultura apresenta foco em resultados e eficácia organizacionais e na execução do trabalho a partir de 
Giuseppe Maria Russo, Patricia Amelia Tomei, Antonio José Braga Linhares \& Andre Moreira Santos

ferramentas apropriadas, pessoas certas e autonomia. Nesse tipo de ambiente é difícil o controle. Em Atena, os meios não são relevantes e sim os resultados. Nesta categoria cultural, a perícia é a base para o poder e a recompensa (remuneração e reconhecimento) é dado aos especialistas.

Por fim, a Cultura Existencial ou Cultura de Pessoa é representada pelo deus grego Dionísio que representa a ideologia existencial. O existencialismo prega sermos responsáveis pelo nosso destino, independente da vontade dos deuses. A Cultura Existencial é encontrada em organizações cujos indivíduos se prendem aos seus valores e são o ponto central da empresa, rejeitando os controles e as metas organizacionais. Existe nesse grupo uma mútua orientação no sentido de todos os membros do grupo seguirem suas próprias direções. Nesse sentido a recompensa depende da competência individual.

Embora as organizações possuam mais de um "deus" (Handy, 1980), as culturas devem apresentar consistência interna, afinal, os indivíduos que estão nas organizações têm influências monoteístas e necessitam de identificação para confrontar com o seu perfil (“deus") predominante.

Neste estudo utilizamos como objeto de correlação com a cultura organizacional um dos sistemas que refletem a cultura organizacional: o de remuneração, que apresenta estratégias específicas. Afinal, Handy (1980, p.9) explica que "diferentes culturas [...] resultam em estilos bem diferentes de administração, estruturas, procedimentos e sistemas de recompensa".

\section{Remuneração e Recompensas}

Ao longo dos últimos 100 anos, as estratégias de remuneração dos empregados vêm sendo modificadas. Nas primeiras décadas do século passado, o pagamento pelo trabalho era com base na produtividade, onde o modelo do homem-econômico, preconizado por Frederick W. Taylor, fundamentou a Teoria Clássica da Administração. Naquela época, existia apenas a remuneração fixa (por jornada ou por peças produzidas) e que não estimulava o aumento da produção, pois não havia o ganho extra para o trabalhador (Hipólito, 2004). Para quebrar o vício da remuneração fixa surgiu a recompensa pelo trabalho realizado, que naquela época era um incentivo pela máxima eficiência (Hipólito, 2004). 
Correlacionando tipos de cultura organizacional com estratégias de remuneração utilizando a tipologia de Charles Handy

Ainda no decorrer da primeira metade do Século 20, surgiu o modelo do homem-social, criado por Elton Mayo, que fundamentou a Teoria das Relações Humanas. Naquele momento, começou a surgir a percepção de "compatibilidade entre o esforço e a recompensa" (Maximiano, 2000, p.248). No entanto, a prática de definir a remuneração com base nos profissionais e sua produção, foi perdendo espaço para o uso do conceito de cargos como principal variável da remuneração. Nesse modelo, o cargo passou a ser utilizado para fixar o salário e os demais rendimentos decorrentes do mesmo. O princípio por trás dessa mudança foi o entendimento de que a remuneração por cargos seria imparcial (meritocrática). No modelo por cargos, a contribuição pessoal não era utilizada como a referência, somente o cargo. Com isso, a recompensa deixou de figurar como motivadora da produção.

Apesar das mudanças ocorridas a partir da década de 60 no mundo, em geral, e das preocupações sobre modelos de recompensas, em particular, na prática, o sistema de recompensas permaneceu adormecido em função do excesso de conservadorismo (Hipólito, 2004). Entretanto, a grande mudança daquela época foi passar a alocar uma importância maior aos aspectos não monetários do que vinha sendo feito, "embora prevalecesse à dificuldade de atribuir recompensas não associadas a aspectos financeiros e a manutenção da remuneração atrelada ao cargo" (Hipólito, 2004, p.28).

Com o surgimento da Teoria Contingencial, o trabalhador assumiu uma posição central e passou a participar como agente de mudanças. Com isso, pesquisas sobre cultura organizacional, liderança, qualidade e melhorias de processos passaram a ocupar posição de destaque nas agendas dos pesquisadores. Assim, na década de 80, começaram a surgir estudos para verificar a influência da remuneração no desempenho organizacional (Lawler, 1981).

Foi a partir do final da década de 80 que novos modelos de remuneração começaram a ser analisados. Como alternativa ao paradigma do cargo e alterando o foco do cargo para a pessoa, surgiram sistemas de remuneração que visavam à superação das desvantagens da abordagem tradicional. Surgia, naquela época, a remuneração por habilidades, aplicada principalmente nas áreas operacionais, altamente especializadas (Lawler, 1990). Aquele modelo buscou resgatar a recompensa por pessoa, tentando se desvincular do modelo de cargos. O pagamento por habilidade reconhecia o grau de capacitação dos empregados ou, como apresenta Milkovich e Newman (1987), fazia com que os empregados recebessem pelas 
Giuseppe Maria Russo, Patricia Amelia Tomei, Antonio José Braga Linhares \& Andre Moreira Santos

habilidades que possuíam em relação ao trabalho (o que eles podem fazer), ao invés do pagamento pelo cargo específico (o que eles estão fazendo).

A partir desta evolução, a remuneração funcional começou a perder força (apesar de estar presente ainda hoje em muitas empresas), buscando atender as mudanças e necessidades organizacionais. Entretanto, com a dificuldade de associar a aquisição de habilidades ao aumento de desempenho daqueles profissionais, cuja contribuição era difícil de mensurar e prever, a mudança sofreu muitas críticas. Para atenuá-las, no início da década de 90 , surgiu a gestão por competências.

Apesar de todas as recentes mudanças, a essência da elaboração de uma estratégia de remuneração não se alterou significativamente e o conceito ainda pode ser considerado como a utilização planejada de um sistema de pagamentos, como mecanismo essencial de integração, com o qual o esforço de várias subunidades ou profissionais é direcionado ao atingimento dos objetivos estratégicos de uma organização (Gomez-Mejia \& Balkin, 1992).

Gomez-Mejia (1992), com base em uma criteriosa revisão da literatura, identificou alguns dos componentes da remuneração: habilidades individuais ou avaliação do cargo; ênfase no longo prazo ou no curto prazo; desempenho organizacional ou da unidade; preocupação com a equidade interna ou externa; medidas de desempenho quantitativas ou qualitativas; a estrutura e o desenho do pacote de remuneração (variáveis como, nível de pagamento da empresa versus mercado; salário fixo ou incentivo; frequência de aumentos e bônus etc.); políticas e procedimentos que regulam o sistema de pagamentos (como, centralização ou descentralização das políticas de pagamento, abertura ou não das informações de pagamento, burocracia ou flexibilidade nas políticas de pagamento etc.).

Heneman, Fisher e Dixon (2001) também detalham os componentes do sistema de remuneração nas seguintes dimensões: forma de recompensa (monetária ou não-monetária); unidade de análise (pessoa ou cargo); base de comparação (interna ou externa); medidas de recompensa (comportamentos ou resultados); nível de recompensa (individual ou unidade de negócios); aumento salarial (fixo ou variável); nível de administração (centralizado ou descentralizado); tempo da implantação (pioneiro ou seguidor); comunicação (aberta ou fechada). 
Correlacionando tipos de cultura organizacional com estratégias de remuneração utilizando a tipologia de Charles Handy

Vale ressaltar que as recompensas intrínsecas, apontadas por Camara (2000), consistem na satisfação gerada pelo próprio trabalho e são as que de fato geram motivação e ajudam a criar vínculos e identidade com a organização. Poderiam ser classificados como mecanismos de recompensas intrínsecas: políticas de reconhecimento, estilo de gestão, estrutura organizacional, clima organizacional e oportunidades de crescimento.

Resumidamente, de acordo com a revisão da literatura (Gomez-Mejia, 1992; Heneman et al., 2001; Camara, 2000; Plothow, 2006; Hipólito, 2004), os principais componentes de remuneração e o construto da estratégia de remuneração deste estudo, estão descritos no Quadro 1.

\section{Quadro 1: Construto da Remuneração}

\begin{tabular}{|c|c|c|c|}
\hline Componente & $\begin{array}{l}\text { Variável } \\
\end{array}$ & Indicador & Autor \\
\hline $\begin{array}{l}\text { Salário-base (SAL) } \\
\text { Constitui-se da remuneração } \\
\text { recebida pelo profissional. }\end{array}$ & $\begin{array}{l}\text { Cargo (nível de responsabilidades } \\
\text { exigidas, etc.) versus Fatores pessoais } \\
\text { (desempenho, tempo de experiência, etc.) }\end{array}$ & $\begin{array}{l}\text { - Cargo } \\
\text { - Pessoa }\end{array}$ & $1,2,3$ \\
\hline $\begin{array}{l}\text { Critérios de avaliação da } \\
\text { contribuição individual (AVA) } \\
\text { Forma como a organização avalia o } \\
\text { desempenho individual. }\end{array}$ & $\begin{array}{l}\text { Senioridade (nível de experiência do } \\
\text { indivíduo) versus } \text { Desempenho } \\
\text { (resultados obtidos). }\end{array}$ & $\begin{array}{l}\text { - Senioridade } \\
\text { - Desempenho }\end{array}$ & 3 \\
\hline Indicadores de desempenho (IND) & $\begin{array}{l}\text { Grupo (equipes ou organização) versus } \\
\text { Indivíduo }\end{array}$ & $\begin{array}{l}\text { - Individual } \\
\text { - Coletivo }\end{array}$ & $1,2,3$ \\
\hline $\begin{array}{l}\text { Natureza dos indicadores (NAT) } \\
\text { Podem ter caráter financeiro ou não- } \\
\text { financeiro }\end{array}$ & $\begin{array}{l}\text { Financeiros (lucro, vendas brutas, etc.) } \\
\text { versus não-financeiros (market share, } \\
\text { nível de reclamação de clientes, etc.). }\end{array}$ & $\begin{array}{l}\text { - Resultados } \\
\text { financeiros } \\
\text { - Resultados não } \\
\text { financeiros } \\
\end{array}$ & 1,3 \\
\hline $\begin{array}{l}\text { Dificuldade das metas (MET) } \\
\text { Indica o quanto a remuneração } \\
\text { variável é de fato. }\end{array}$ & Difíceis versus Fáceis & $\begin{array}{l}\text { - Fáceis } \\
\text { - Difíceis }\end{array}$ & 3 \\
\hline $\begin{array}{l}\text { Recompensas (REC) } \\
\text { Mensura a forma como são } \\
\text { recompensados os profissionais pelo } \\
\text { seu desempenho ou senioridade. }\end{array}$ & $\begin{array}{l}\text { Financeira (em espécie, em ações, etc.) } \\
\text { versus Não-financeiras (benefícios, } \\
\text { placas, etc.). }\end{array}$ & $\begin{array}{l}\text { - Financeira } \\
\text { - Não-financeira }\end{array}$ & $\begin{array}{l}1,2 \\
3,5\end{array}$ \\
\hline $\begin{array}{l}\text { Clima Organizacional (CLI) } \\
\text { É uma recompensa intrínseca do } \\
\text { dia-a-dia de trabalho. Quanto } \\
\text { melhor o clima organizacional, mais } \\
\text { aderente o profissional fica à } \\
\text { organização. }\end{array}$ & Excelente versus Péssimo & $\begin{array}{l}\text { - Excelente } \\
\text { - Ruim }\end{array}$ & 4 \\
\hline $\begin{array}{l}\text { Oportunidade de crescimento e } \\
\text { desenvolvimento (OPO) } \\
\text { É uma recompensa intrínseca que } \\
\text { favorece a permanência do } \\
\text { indivíduo na organização. }\end{array}$ & Abundante versus Escassa & $\begin{array}{l}\text { - Abundante } \\
\text { - Escassa }\end{array}$ & 4 \\
\hline $\begin{array}{l}\text { Nível de remuneração (REM) } \\
\text { Mede a competitividade da } \\
\text { organização frente as suas } \\
\text { concorrentes e demais empresas do } \\
\text { mercado. }\end{array}$ & Total versus Mercado & $\begin{array}{l}\text { - Abaixo da } \\
\text { mediana de } \\
\text { mercado } \\
\text { - Acima da } \\
\text { mediana de } \\
\text { mercado }\end{array}$ & 3 \\
\hline
\end{tabular}


Giuseppe Maria Russo, Patricia Amelia Tomei, Antonio José Braga Linhares \& Andre Moreira Santos

\begin{tabular}{|l|l|l|l|}
\hline $\begin{array}{l}\text { Equidade (EQU) } \\
\text { Analisa o equilíbrio entre a } \\
\text { manutenção da equidade interna e } \\
\text { externa. }\end{array}$ & $\begin{array}{l}\text { Interna (cargos semelhantes têm } \\
\text { remuneração semelhante) versus Externa } \\
\text { (empresas e segmentos semelhantes } \\
\text { remuneram cargos equivalentes de forma } \\
\text { similar). }\end{array}$ & $\begin{array}{l}\text { - Interna } \\
\text { Externa }\end{array}$ & $1,2,3$ \\
\hline $\begin{array}{l}\text { Composição (COM) } \\
\text { Distribuição das recompensas. }\end{array}$ & $\begin{array}{l}\text { Fixa (salário, 13 }{ }^{\circ} \text {, etc.) versus Variável } \\
\text { (bônus, remuneração variável de vendas, } \\
\text { etc.). }\end{array}$ & $\begin{array}{l}\text { - Fixo } \\
\text { - Variável }\end{array}$ & 1,3 \\
\hline $\begin{array}{l}\text { Tempo (TEM) } \\
\text { Refere-se a diferença para o } \\
\text { profissional receber hoje ou } \\
\text { amanhã, do ponto de vista de fluxo } \\
\text { de caixa. }\end{array}$ & $\begin{array}{l}\text { Curto Prazo versus Longo Prazo. O } \\
\text { tempo é uma variável que auxilia na } \\
\text { retenção dos profissionais, pois coloca } \\
\text { prazos e/ou barreiras financeiras de } \\
\text { saídas. }\end{array}$ & $\begin{array}{l}\text { - Curto prazo } \\
\text { - Longo prazo }\end{array}$ & 1,5 \\
\hline
\end{tabular}

Nota: 1- Gomez-Mejia (1992); 2- Heneman et al. (2001); 3- Plothow (2006); 4- Camara (2000); 5- Hipólito (2004).

Fonte: os autores

Elaborar uma estratégia de remuneração, decidindo sobre cada um destes componentes, busca estimular comportamentos positivos, visando o alcance dos objetivos organizacionais (O’Neal, 1992). Porém, o alinhamento da remuneração com os objetivos estratégicos não é simples, constituindo-se, de acordo com Lawler (1990), um dos maiores desafios das organizações. Marx e Soares (2008, p.26), por exemplo, não conseguiram comprovar de forma empírica "a difusão da lógica da geração de valor ao acionista no ambiente de produção", considerando nesta análise a utilização da remuneração e do processo de avaliação de desempenho como ferramentas.

Silva (2008) afirma que o sistema de recompensas de uma organização deve assumir como objetivos: atrair, reter e motivar empregados de alto potencial; reforçar a cultura da empresa e o modelo estrutural dela emergente; realizar os objetivos anteriores ao menor custo possível. Da mesma forma, Barton (2006), enfatiza que os programas de recompensa contribuem para o direcionamento da cultura organizacional. Nesse sentido, a remuneração deve estar alinhada a cultura organizacional.

Portanto, definir uma estratégia de remuneração compreende identificar os componentes (vide Quadro 1) que devem compor o portfólio de remuneração da organização, dentro de contextos culturais específicos que, certamente, irão variar entre as organizações, dependendo da cultura organizacional.

\section{Inter-relação dos construtos}


Correlacionando tipos de cultura organizacional com estratégias de remuneração utilizando a tipologia de Charles Handy

De acordo com o referencial a inter-relação entre os tipos de cultura de Handy (1980) e os construtos das estratégias de remuneração, apresenta-se conforme descrito no Quadro 2.

Quadro 2: Inter-relação entre as Variáveis Culturais e as Estratégias de Remuneração

\begin{tabular}{|c|c|c|c|c|}
\hline Construtos & $\begin{array}{c}\text { Zeus } \\
\text { Foco no relaciona- } \\
\text { mento individual. }\end{array}$ & $\begin{array}{c}\text { Apolo } \\
\text { Foco nos papéis e } \\
\text { nas regras. } \\
\end{array}$ & $\begin{array}{c}\text { Atena } \\
\text { Foco nos resultados } \\
\text { e na eficácia. }\end{array}$ & $\begin{array}{c}\text { Dionísio } \\
\begin{array}{c}\text { Foco na individuali- } \\
\text { dade. }\end{array}\end{array}$ \\
\hline SAL & Ênfase na pessoa. & Ênfase no cargo. & Ênfase na pessoa. & Ênfase na pessoa. \\
\hline AVA & $\begin{array}{l}\text { Ênfase na } \\
\text { senioridade. }\end{array}$ & Variável. & $\begin{array}{l}\text { Ênfase no desem- } \\
\text { penho. }\end{array}$ & $\begin{array}{l}\text { Ênfase no desem- } \\
\text { penho. }\end{array}$ \\
\hline IND & $\begin{array}{l}\text { Ênfase na individua- } \\
\text { lidade. }\end{array}$ & Variável. & Variável. & $\begin{array}{l}\text { Ênfase na } \\
\text { individualidade. }\end{array}$ \\
\hline NAT & $\begin{array}{l}\text { Ênfase nos fatores } \\
\text { comportamentais. }\end{array}$ & Variável. & Ênfase no financeiro. & Variável. \\
\hline MET & Variável. & Variável. & $\begin{array}{l}\text { Ênfase nas metas } \\
\text { difíceis. }\end{array}$ & Difícil. \\
\hline REC & Variável. & Ênfase no financeiro. & Ênfase no financeiro. & Variável. \\
\hline CLI & Variável. & Variável. & $\begin{array}{l}\text { Ênfase no Ruim pela } \\
\text { pressão. }\end{array}$ & Ênfase no excelente. \\
\hline OPO & Variável. & Variável. & $\begin{array}{l}\text { Ênfase no abundan- } \\
\text { tes. }\end{array}$ & $\begin{array}{l}\text { Ênfase no abundan- } \\
\text { tes. }\end{array}$ \\
\hline REM & Variável. & Variável. & $\begin{array}{l}\text { Ênfase na remunera- } \\
\text { ção acima da media- } \\
\text { na no variável. }\end{array}$ & $\begin{array}{l}\text { Ênfase na remunera- } \\
\text { ção acima da media- } \\
\text { na. }\end{array}$ \\
\hline QUE & $\begin{array}{l}\text { Ênfase no equilíbrio } \\
\text { interno. }\end{array}$ & $\begin{array}{l}\text { Ênfase no equilíbrio } \\
\text { interno. }\end{array}$ & Variável. & $\begin{array}{l}\text { Ênfase no equilíbrio } \\
\text { externo. }\end{array}$ \\
\hline COM & Variável. & $\begin{array}{l}\text { Ênfase na remunera- } \\
\text { ção fixa. }\end{array}$ & $\begin{array}{l}\text { Ênfase na remunera- } \\
\text { ção variável. }\end{array}$ & Variável. \\
\hline TEM & $\begin{array}{l}\text { Ênfase no longo } \\
\text { prazo. }\end{array}$ & $\begin{array}{l}\text { Ênfase no longo } \\
\text { prazo. }\end{array}$ & $\begin{array}{l}\text { Ênfase no curto } \\
\text { prazo. }\end{array}$ & $\begin{array}{l}\text { Ênfase no curto } \\
\text { prazo. }\end{array}$ \\
\hline
\end{tabular}

Fonte: os autores

\section{PROCEDIMENTOS METODOLÓGICOS}

Entendendo a cultura organizacional como um conjunto de valores compartilhados entre os membros da organização e sabendo que existem diversas tipologias para identificar a cultura organizacional, este estudo optou pela aplicação do construto de Handy (1980). Para a identificação da estratégia de remuneração adotamos o construto desenvolvido neste estudo.

\section{Universo e amostra}

O universo para a aplicação dos construtos de cultura e remuneração, apresentados no referencial teórico, é composto por 76 empresas associadas ao Grupo de Permuta de Informações Salariais (GRUPISA). O GRUPISA é a maior entidade sem fins lucrativos de remuneração da América do Sul que congrega empresas de todos os setores e tamanhos com o 
Giuseppe Maria Russo, Patricia Amelia Tomei, Antonio José Braga Linhares \& Andre Moreira Santos

objetivo de realizar pesquisas, desenvolver estudos e compartilhar informações na área de remuneração (GRUPISA, 2009). O tamanho da amostra foi calculado a partir da fórmula de Rea e Parker (1997), apropriada para pequenas populações. Para um intervalo de confiança de 95\%, ou seja, um grau de significância de 5\%, a amostra foi de 46 empresas, com uma margem de erro de $5 \%$. Os sujeitos escolhidos para participar da pesquisa foram os executivos seniores destas empresas.

\section{Seleção dos sujeitos}

Para avaliação dos construtos da pesquisa foram selecionados os executivos seniores da área de remuneração de cada uma das empresas da amostra. Avaliar o desempenho de alguma atividade organizacional com um único respondente vem sendo utilizado na literatura (Birkinshaw, Hood, \& Jonsson, 1998; Delery \& Doty, 1996; Geringer \& Hebert, 1989; Lee \& Beamish, 1995; Shaw, Delery, Jenkins, \& Gupta, 1998), assim como, em estudos de cultura organizacional e remuneração (Bourantas et al., 1990; Balkin \& Gomez-Mejia, 1987). De acordo com Fey e Denison (2003) os estudos com um único respondente, publicados nas melhores revistas científicas, são citados frequentemente, sugerindo que quando adequadamente executado seus resultados são aceitos. Além disso, há bons precedentes para se utilizar medidas de avaliação de percepção com um único respondente (Tosi \& GomezMejia, 1994; Delaney \& Huselid, 1996; Denison \& Mishra, 1995), principalmente quando o tema pesquisado depende de profundo conhecimento de um especialista.

\section{Coleta e tratamento de dados}

Para a coleta de dados junto às empresas em 2010 foi realizado um levantamento de percepções, do tipo survey, com auxílio de um questionário estruturado, construído com base nos construtos e indicadores apresentados no referencial teórico.

O instrumento desenvolvido por Handy (1980) estabelece uma escolha forçada (escala ipsative) multidimensional que reduz a inflação dos escores e apresentam maior validade de critério em comparação à escala Likert (Heggestad, Morrison, Reeve, \& McCloy, 2006). Por outro lado, esse tipo de medida apresenta limitações, pois não fornece dados normativos, como a escala Likert, necessários para a realização de análises estatísticas (Mccloy, Heggestad, \& Reeve, 2005), como a Correlação de Pearson, pois as variáveis não são 
Correlacionando tipos de cultura organizacional com estratégias de remuneração utilizando a tipologia de Charles Handy

estatisticamente independentes (Closs, 1996). A problemática da escolha forçada está pautada na violação de um dos principais pressupostos da teoria clássica dos testes que é a independência da variância do erro, pois a correlação negativa que se aproxima de zero aumenta à medida que aumenta o número de escalas do teste. Além disso, não atende ao critério paramétrico padronizado, pois o nível de medida não é ordinal e nem escalar (Welter \& Capitão, 2007). Por outro lado, a aplicação do instrumento desenvolvido por Handy (1980) se aplicado com o uso da escala de Likert pode apresentar uma tendenciosidade nas respostas criando um viés nos resultados, por permitir ao respondente avaliar as variáveis nos seus limites extremos.

Para possibilitar o uso do instrumento de Handy (1980) nas análises estatísticas paramétricas, foram aplicadas duas escalas simultaneamente (Closs, 1996). Inicialmente, foi solicitado aos sujeitos para responderem as variáveis do instrumento de Handy (1980), conforme orientações do autor, para medir o grau de importância de cada uma delas para cada pergunta. Em seguida, foi solicitado aos sujeitos para avaliar individualmente o grau de intensidade de cada uma das variáveis, usando a escala Likert, graduando de 1 a 5, sendo um baixa intensidade e 5 alta intensidade. Com a ajuda do formulário eletrônico, o grau de intensidade das variáveis marcadas com os graus de importância 1 e 2 somente tinham disponíveis os graus de intensidade 1, 2 e 3; enquanto que as variáveis marcadas com os graus de importância 3 e 4 somente tinham disponíveis os graus de intensidade 3, 4 e 5 .

Antes da aplicação da pesquisa à amostra, o questionário foi submetido a um pré-teste com especialistas no tema remuneração, para verificar sua clareza e sua capacidade de captação dos dados e das informações necessárias para responder às questões. O questionário contendo a tipologia de Handy (1980) não foi validado nesta pesquisa por ser amplamente utilizado em pesquisas acadêmicas (Gainey et al., 1999; Naves \& Coleta, 2003) e em estudos empíricos por Bourantas et al., (1990) e Gomide e Martins (1987).

Foi enviado um e-mail pelo GRUPISA aos gestores seniores de remuneração de cada uma das 76 empresas, fornecendo o endereço eletrônico da pesquisa, disponível na Internet.

Foram realizados testes estatísticos de Correlação de Pearson para verificar o grau de correlação entre as variáveis de remuneração e os tipos de cultura organizacional. A Regressão Múltipla (método stepwise) para identificar os melhores preditores do construto de remuneração para cada um dos quatro tipos de cultura organizacional (Handy, 1980). A 
Giuseppe Maria Russo, Patricia Amelia Tomei, Antonio José Braga Linhares \& Andre Moreira Santos

escolha do método stepwise foi decorrente da sua finalidade ser geralmente utilizada em estudos exploratórios (Abbad \& Torres, 2002). Para evitar qualquer viés nos resultados, os dados foram analisados quanto à existência de outliers e a linearidade do relacionamento das variáveis (Hair, Anderson, Tatham \& Black, 1998). Os resultados da matriz de correlação demonstram as relações entre a tipologia de Handy (1980) e os componentes do construto de remuneração. Quanto menor o p-value (ex.: p $\leq 0,001)$ maior a correlação entre elas.

Objetivando reduzir o viés do resultado da análise, foram adicionadas à regressão múltipla, variáveis de controle que podem causar impacto ou que podem estar correlacionadas com o construto (Cable \& Judge, 1996) de remuneração:

- $\quad$ Tamanho da empresa (Bourantas et al., 1990; Majumdar, 1997): pequena (0) até 100 empregados, média (1) até 500 empregados, e grande (2) acima de 500 empregados.

- $\quad$ Ramo de atividade (Croucher, Gooderham, \& Parry, 2006): indústria (0), comércio (1) e serviços (2).

- $\quad$ Idade da empresa (Majumdar, 1997): número de anos de existência desde a fundação da empresa (operacionalizado com $\log 10$, ver Croucher et al., 2006).

- $\quad$ Origem (Bourantas et al., 1990): nacional (0) e multinacional (1).

- Receita (Conyon, Peck, \& Sadler, 2009): valor do faturamento de vendas anual (operacionalizado com $\log 10$ receita).

Os cálculos estatísticos foram realizados com o software SPSS versão 13.

\section{ANÁLISE E DISCUSSÃO DOS RESULTADOS}

As escalas ipsative e Likert aplicadas na tipologia de Handy (1980) apresentaram resultados proporcionais (Figuras 1 e 2), permitindo o uso dos resultados nas análises estatísticas paramétricas. 
Correlacionando tipos de cultura organizacional com estratégias de remuneração utilizando a tipologia de Charles Handy

Figura 1. Resultado Escala Ipsative

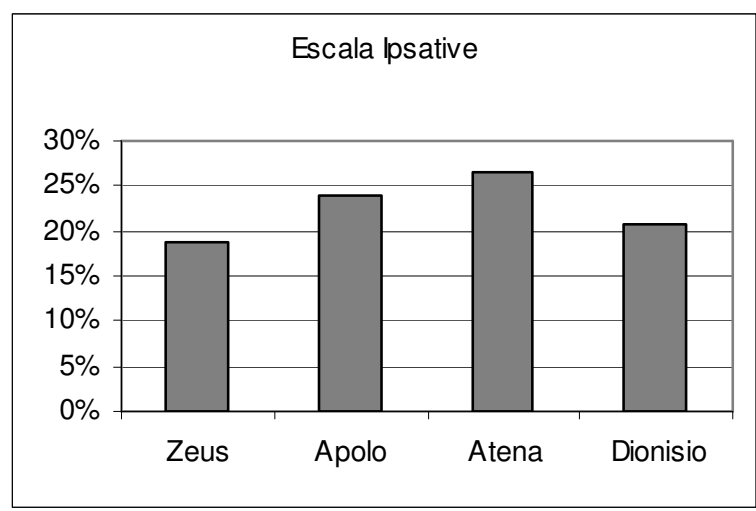

Figura 2. Resultado Escala Likert

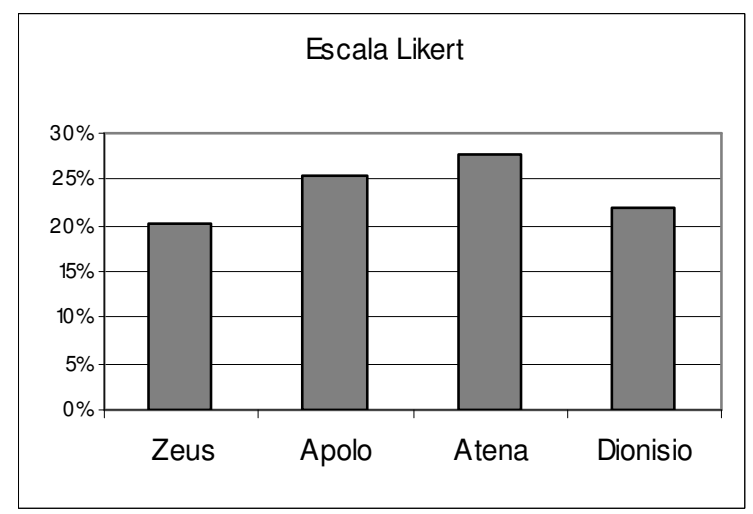

Fonte: os autores

O perfil da amostra de 46 empresas apresentou o resultado detalhado na Tabela 3. Predominantemente, a amostra foi composta por empresas com mais de 500 empregados (80\%), que atuavam na atividade de serviços (59\%), com mais de 25 anos de existência (65\%), com capital nacional $(65 \%)$ e com faturamento de até 500 milhões (43\%). As empresas da amostra são predominantemente maduras, de grande porte e do segmento de serviços.

Quadro 3: Dados Demográficos da Amostra

\begin{tabular}{|c|c|c|c|c|c|c|c|c|c|c|c|c|c|c|c|}
\hline \multirow[t]{2}{*}{ Deuses } & \multicolumn{3}{|c|}{ Empregados } & \multicolumn{3}{|c|}{ Atividade } & \multicolumn{3}{|c|}{ Idade } & \multicolumn{2}{|c|}{ Capital } & \multicolumn{3}{|c|}{ Faturamento } & \multirow{2}{*}{$\frac{\text { Total }}{\text { Deuses }}$} \\
\hline & $\begin{array}{l}\text { Até } \\
100\end{array}$ & $\begin{array}{c}101 \\
\mathrm{a} \\
500\end{array}$ & $\begin{array}{c}+ \\
500\end{array}$ & Ind & Com & Ser & $\begin{array}{c}\text { até } \\
10 \\
\text { anos }\end{array}$ & $\begin{array}{c}11 \mathrm{a} \\
25 \\
\text { anos }\end{array}$ & $\begin{array}{l}+25 \\
\text { anos }\end{array}$ & $\mathrm{Nac}$ & Int & $\begin{array}{c}\text { até } \\
500 \\
\text { MM }\end{array}$ & $\begin{array}{c}501 \\
\mathrm{a} \\
1 \mathrm{bi}\end{array}$ & $\begin{array}{c}+ \\
1 \mathrm{bi}\end{array}$ & \\
\hline Zeus & 0 & 2 & 3 & 2 & 1 & 2 & 1 & 0 & 4 & 1 & 4 & 2 & 1 & 2 & 5 \\
\hline Apolo & 0 & 1 & 8 & 2 & 0 & 7 & 2 & 0 & 7 & 6 & 3 & 2 & 3 & 4 & 9 \\
\hline Atena & 0 & 6 & 21 & 4 & 7 & 16 & 4 & 7 & 16 & 18 & 9 & 15 & 5 & 7 & 27 \\
\hline Dionisio & 0 & 0 & 5 & 0 & 3 & 2 & 2 & 0 & 3 & 5 & 0 & 1 & 2 & 2 & 5 \\
\hline Subtotal & 0 & 9 & 37 & 8 & 11 & 27 & 9 & 7 & 30 & 30 & 16 & 20 & 11 & 15 & - \\
\hline $\mathrm{N}$ & & 46 & & & 46 & & & 46 & & & & & 46 & & 46 \\
\hline$\%$ & 0 & 20 & 80 & 17 & 24 & 59 & 20 & 15 & 65 & 65 & 35 & 43 & 24 & 33 & - \\
\hline $\mathrm{N}$ & & $100 \%$ & & & $100 \%$ & & & $100 \%$ & & & & & $100 \%$ & & $100 \%$ \\
\hline
\end{tabular}

Notas: Empregados na empresa (até 100, de 101 a 500, mais de 500); Atividade (indústria, comércio, serviços); Idade da empresa (até 10 anos, entre 11 e 25 anos, mais de 25 anos); Capital da empresa (nacional, internacional); Faturamento da empresa (até 500 milhões, de 501 milhões a 1 bilhão, mais de 1 bilhão).

Fonte: os autores

A maioria das empresas apresentou o tipo de cultura denominada Atena que tem foco, principalmente, nos resultados e na eficácia e dá ênfase aos fatores pessoais, as metas com alto grau de dificuldade, as recompensas financeiras, ao clima organizacional com conflito, as oportunidades de crescimento e desenvolvimento abundantes, ao nível de remuneração acima 
Giuseppe Maria Russo, Patricia Amelia Tomei, Antonio José Braga Linhares \& Andre Moreira Santos

da mediana de mercado, principalmente no composto variável, e a visão de curto prazo. As empresas do tipo Atena apresentam a Cultura de Tarefa que objetiva a execução do trabalho com as pessoas certas, onde os meios não são relevantes e sim os resultados e onde o reconhecimento é dado aos especialistas.

A Correlação de Pearson apresentou escores significantes, com valores médios entre 0,30 e 0,46 (Cohen, 1988), conforme detalhado na Tabela 4. O teste de Kolmogorov-Smirnov apresentou resultados não-significativos $(\mathrm{p}>0,05)$ para as variáveis de remuneração e cultura organizacional, indicando normalidade (Figueiredo \& Silva, 2009).

Tabela 1: Resultado da Correlação de Pearson

\begin{tabular}{lcccccccccccc}
\hline & SAL & AVA & IND & NAT & MET & REC & CLI & OPO & REM & EQU & COM & TEM \\
\hline Zeus & 0,28 & $-0,13$ & $-0,20$ & $0,38^{*}$ & $-0,21$ & $0,38^{*}$ & $0,37^{*}$ & 0,01 & 0,02 & 0,14 & $-0,28$ & $-0,25$ \\
\hline Apolo & 0,03 & $-0,09$ & $-0,03$ & 0,10 & 0,20 & $-0,04$ & $-0,02$ & $0,46^{*}$ & $-0,11$ & $0,31^{*}$ & $-0,02$ & $-0,04$ \\
\hline Atena & 0,12 & 0,13 & 0,04 & $-0,13$ & 0,05 & $-0,27$ & $-0,19$ & $0,30^{*}$ & 0,08 & 0,11 & $0,30^{*}$ & $0,33^{*}$ \\
\hline Dionísio & $-0,02$ & 0,22 & $-0,08$ & 0,12 & $-0,33^{*}$ & 0,04 & $-0,11$ & $-0,31 *$ & $0,31 *$ & $-0,21$ & 0,01 & 0,12 \\
\hline $\mathrm{N}=$ & 46 & 46 & 46 & 46 & 46 & 46 & 46 & 46 & 46 & 46 & 46 & 46
\end{tabular}

Notas: 46 empresas. SAL: salário-base; AVA: critério de avaliação; IND: indicadores de desempenho; NAT: natureza dos indicadores; MET: dificuldade das metas; REC: recompensas; CLI: clima organizacional; OPO: oportunidades de crescimento e desenvolvimento; REM: nível de remuneração; QUE: equidade da remuneração; COM: composição da remuneração; TEM: tempo de remuneração.

* valores significativos $\mathrm{p}<0.05$.

Fonte: os autores

Todas as relações de linearidade significantes indicam que as estratégias de remuneração adotadas pelas organizações da amostra atendem aos conceitos dos tipos de cultura de Handy (1980) (veja Quadro 2).

Pode-se verificar, a partir dos resultados da Tabela 4, que as variáveis que apresentaram uma relação de linearidade direta com o tipo de cultura Zeus foram a natureza dos indicadores (NAT), as recompensas (REC) e o clima organizacional (CLI). Com o tipo de cultura Apolo foram as oportunidades de crescimento e desenvolvimento (OPO) e a equidade da remuneração (EQU). Com o tipo de cultura Atena as variáveis foram as oportunidades de crescimento e desenvolvimento (OPO), o composição da remuneração $(\mathrm{COM})$ e o tempo de remuneração (TEM). E, por último, com o tipo de cultura Dionísio as variáveis foram a dificuldade das metas (MET), as oportunidades de crescimento e desenvolvimento (OPO) e o nível de remuneração (REM). 
Correlacionando tipos de cultura organizacional com estratégias de remuneração utilizando a tipologia de Charles Handy

No que se refere as correlações do tipo cultural Zeus percebemos a relevância dos relacionamentos com ênfase nos fatores comportamentais como indicadores para recompensas e remuneração. Também apresenta ênfase nas recompensas, seguindo um modelo de premiar aqueles que estão mais próximos da liderança. Além disso, ressalta a necessidade da gestão do clima organizacional que pode provocar impacto positivo ou negativo, dependendo do grau de relação entre lideres e liderados.

Nas correlações do tipo cultura Apolo a ênfase nas regras e nas normas favorece que os profissionais tenham maior clareza sobre as oportunidades de desenvolvimento e crescimento na carreira. As oportunidades estão disponíveis para os profissionais que atendam aos prérequisitos especificados nos procedimentos e normas organizacionais. A correlação com a equidade demonstra a busca de Apolo tanto pelo equilíbrio interno da remuneração entre os profissionais com o mesmo grau de complexidade em suas funções.

O tipo de cultura Atena, no que se refere a correlação com a variável oportunidades de crescimento e desenvolvimento, favorece a busca de resultados, pois não existem barreiras para o crescimento dos profissionais, quando eles alcançam os objetivos organizacionais. A composição da remuneração também é importante para a cultura Atena, tendo em vista que desenvolve estratégias de remuneração atraentes aos profissionais e que visam demonstrar a distribuição dos ganhos gerados pelo alcance dos resultados. A variável tempo demonstra a viabilidade de ganhos rápidos, aumentando a busca por resultados imediatos.

As correlações negativas no tipo de cultura Dionísio reforçam o foco na individualidade dos profissionais, tanto na variável dificuldade das metas quanto na oportunidade de crescimento e desenvolvimento. Quanto às metas os profissionais presentes na cultura Dionísio preferem metas difíceis devido ao alto grau de especialização. As oportunidades de crescimento e desenvolvimento são percebidas como escassas, pois os profissionais nesta cultura buscam ao máximo a customização de seu composto de remuneração e sua carreira na organização. No que refere a variável nível de remuneração, a correlação é positiva, demonstrando a relevância da competitividade de remuneração dos profissionais da cultura Dionísio em relação ao mercado. Profissionais com características diferenciadas demandam estratégias de remuneração diferenciadas. 
Giuseppe Maria Russo, Patricia Amelia Tomei, Antonio José Braga Linhares \& Andre Moreira Santos

Os resultados da análise de regressão múltipla stepwise (Tabela 5) demonstram os preditores de cada um dos tipos de cultura organizacional.

O modelo calculado para verificar a explicação provida pelas estratégias de remuneração sobre o tipo de cultura organizacional Zeus, revela que três variáveis de remuneração conseguem explicar em conjunto $42,0 \%$ da variância do tipo de cultura. O peso dos preditor positivo REC (financeira e não-financeira), contribuindo com $41,7 \%$ na equação, revela que a recompensa dos profissionais pelo desempenho ou senioridade é necessária, podendo variar entre uma estratégia pecuniária (em espécie, em ações, etc.) ou não-financeira (benefícios, cartas, medalhas, placas, etc.). Este resultado reforça as estratégias apresentadas pelos estudos de Gomez-Mejia (1992), Heneman et al. (2001), Plothow (2006) e Hipólito (2004). Heneman et al. (2001), principalmente, expressa a importância da aplicação da remuneração financeira ou não-financeira com estratégia de remuneração. Da mesma forma, o peso do preditor positivo NAT (fatores econômicos e comportamentais), contribuindo com 34,0\% na equação, aponta que os indicadores têm ênfase nos fatores comportamentais, diretamente relacionado a liderança de Zeus e ao comprometimento dos empregados. Este resultado contribui para as estratégias apresentadas por Plothow (2006) e Gomez-Mejia (1992). Para este último, e essencial a aplicação das estratégias de remuneração quantitativas e qualitativas.

Tabela 5: Resultado da Regressão Múltipla Stepwise

\begin{tabular}{|c|c|c|c|c|c|}
\hline $\begin{array}{c}\text { Variável } \\
\text { Dependente }\end{array}$ & Variável Independente & $\begin{array}{c}\text { Coeficiente } \\
\text { Padronizado Beta } \\
\end{array}$ & Sig. & $\mathbf{R}$ & $\mathbf{R}^{2}$ \\
\hline \multirow{4}{*}{ Zeus } & Recompensas (REC) & 0,417 & 0,002 & \multirow{4}{*}{0,648} & \multirow{4}{*}{0,420} \\
\hline & Idade da empresa (IDA) & 0,340 & 0,010 & & \\
\hline & Natureza dos Indicadores (NAT) & 0,308 & 0,018 & & \\
\hline & Tempo de Remuneração (TEM) & $-0,270$ & 0,036 & & \\
\hline Apolo & Oportunidades (OPO) & 0,458 & 0,002 & 0,458 & 0,210 \\
\hline \multirow{2}{*}{ Atena } & Tempo de Remuneração (TEM) & 0,357 & 0,015 & \multirow{2}{*}{0,447} & \multirow{2}{*}{0,200} \\
\hline & Recompensas (REC) & $-0,299$ & 0,039 & & \\
\hline \multirow{3}{*}{ Dionísio } & Dificuldade das Metas (MET) & $-0,273$ & 0,031 & \multirow{3}{*}{0,671} & \multirow{3}{*}{0,450} \\
\hline & Nível de Remuneração (REM) & 0,415 & 0,001 & & \\
\hline & Equidade da Remuneração (EQU) & $-0,441$ & 0,003 & & \\
\hline
\end{tabular}

Notas: 46 empresas.

Fonte: os autores

Por fim, o peso do preditor negativo TEM (curto e longo prazos), contribuindo negativamente com $27,0 \%$ na equação, revela que apesar da prática da maioria das empresas da amostra estar voltada para o curto prazo, o esperado seria o contrário, conforme apresentado no referencial teórico (ênfase no longo prazo). Este resultado contraria as estratégias apresentadas pelos estudos de Gomez-Mejia (1992) e Hipólito (2004) quanto a importância da aplicação das 
Correlacionando tipos de cultura organizacional com estratégias de remuneração utilizando a tipologia de Charles Handy

estratégias de remuneração.. A idade da empresa também despontou como preditor positivo do tipo de cultura Zeus, contribuindo com 34,0\% na equação, ratificando o referencial teórico de que o tempo de relacionamento entre Zeus e seus súditos é desejável.

Para o tipo de cultura Apolo houve apenas uma variável da estratégia de remuneração como preditor, explicando $21,0 \%$ da variância do tipo de cultura. O preditor positivo OPO, contribuindo com 45,8\% na equação, revela que em uma cultura na qual as regras são claras, os empregados têm perfeita visualização das oportunidades de crescimento e isso é um fator de retenção dos mesmos. Este resultado reforça as estratégias apresentadas pelos estudos de Camara (2000).

Para o modelo calculado para verificar a explicação provida pelas estratégias de remuneração sobre o tipo de cultura organizacional Atena, duas variáveis de remuneração conseguem explicar em conjunto $20,0 \%$ da variância. O peso do preditor positivo TEM (curto e longo prazos), contribuindo com $35,7 \%$ na equação, revela que a retenção do empregado tem ênfase no curto prazo, confirmando o referencial teórico, para a cultura Atena. Este resultado reforça as estratégias apresentadas pelos estudos de Gomez-Mejia (1992) e Hipólito (2004). Da mesma forma, o peso do preditor negativo REC (financeira e não-financeira), contribuindo negativamente com 29,9\% na equação, aponta que o resultado da amostra pode variar entre uma estratégia pecuniária (em espécie, em ações, etc.) ou não-financeira (benefícios, cartas, medalhas, placas, etc.), apesar do referencial teórico indicar que a recompensa dos profissionais tem ênfase no financeiro Este resultado contraria as estratégias apresentadas pelos estudos de Gomez-Mejia (1992), Heneman et al. (2001), Plothow (2006) e Hipólito (2004). Este resultado pode representar um modelo predominantemente adotado pelas empresas no Brasil (Barros, 2003).

Para o tipo de cultura Dionísio três variáveis de remuneração despontaram como preditor, explicando em conjunto $45,0 \%$ da variância do tipo de cultura. O preditor negativo MET (difíceis e fáceis), contribuindo com $27,3 \%$ na equação, reitera que os profissionais daquelas organizações preferem metas organizacionais difíceis, pois gostam de ser desafiados devido ao alto grau de especialização profissional e por se sentirem exclusivos (exemplo: publicitários, em agências e médicos especialistas, em hospitais). Este resultado contraria a estratégia apresentada por Plothow (2006). As empresas da amostra demonstram práticas voltadas quase que exclusivamente para metas difíceis. 
Giuseppe Maria Russo, Patricia Amelia Tomei, Antonio José Braga Linhares \& Andre Moreira Santos

Por outro lado, o peso do preditor positivo REM (abaixo ou acima da mediana de mercado), contribuindo com $41,5 \%$ na equação, aponta que as empresas da amostra têm ênfase na remuneração acima da mediana, decorrente do prêmio pago aos especialistas. De forma inversa, este resultado contribui para a estratégia apresentada por Plothow (2006). De forma contrária, o peso do preditor EQU (interno e externo), contribuindo negativamente com $44,1 \%$ na equação, demonstra que apesar da prática das empresas da amostra estar voltada para o interno, o esperado seria o inverso, conforme apresentado no referencial teórico (ênfase no mercado externo). Ou seja, as empresas com o tipo de cultura Dionísio precisam se assegurar que a remuneração dos profissionais especialistas esteja compatível com os especialistas do mercado, para não correr o risco de perder talentos.

As divergências com a literatura podem ter motivo na dificuldade das empresas se posicionarem em pólos extremos nas estratégias de remuneração, buscando posicionamentos intermediários (Gomez-Mejia, 1992). Outro fator que pode justificar algumas correlações negativas, em especial para as variáveis de estratégia de remuneração visitadas por Plothow (2006), é o modelo cultural nacional que difere do americano (Barros, 2003). Além disso, adotar uma estratégia de remuneração em detrimento de outra pode ser função do momento da empresa. Fatores como o ciclo de vida do produto, o tamanho da empresa e as características de mercado e do negócio podem influenciar nas decisões acerca da estratégia de remuneração (Moreira, 2008).

\section{CONCLUSÃO}

Este estudo procurou identificar as correlações entre os tipos de cultura organizacional propostos por Handy (1980) e as estratégias de remuneração, estudadas na literatura acadêmica e também as variáveis de remuneração preditoras dos tipos de cultura organizacional de Handy (1980).

Concluímos que as variáveis de remuneração impactam de forma diferente cada um dos tipos de cultura organizacional. Este resultado reforça a teoria quanto ao fato de que culturas diferentes definem estratégias organizacionais diferentes (Tomei et al., 2008). Nesse sentido, as estratégias de remuneração podem explicar os tipos de cultura organizacional. Relacionar os tipos de cultura organizacional e as estratégias de remuneração amplia o campo de 
Correlacionando tipos de cultura organizacional com estratégias de remuneração utilizando a tipologia de Charles Handy

investigação e contribui, de forma significativa, para as organizações alinharem suas estratégias à cultura organizacional, podendo promover uma forma de retenção dos seus profissionais (Schiehll \& Morissette, 2000).

Os gestores organizacionais que planejam no longo prazo, devem buscar identificar a cultura organizacional para proporcionar o alinhamento cultural dos seus profissionais com a aplicação de práticas que estimulem o alto desempenho. Organizações com a cultura Zeus devem buscar enaltecer os relacionamentos entre a liderança e seus profissionais, dando ênfase aos fatores comportamentais em todas as esferas do sistema de remuneração (GomezMejia, 1992). Além disso, sugerimos que os relacionamentos sejam mantidos com visão de longo prazo, pois o tempo é uma variável relevante (Heneman et al. 2001). Para as organizações com a cultura Apolo a ênfase deve ser aplicada nas oportunidades de crescimento e desenvolvimento, pois as oportunidades de crescimento constituem uma forma de recompensa intrínseca que favorece a permanência do profissional na organização (Camara, 2000). Nas organizações com a cultura Atena o foco deve ser nas recompensas ao longo do tempo, em especial as financeiras, como fator motivador (Gomez-Mejia, 1992; Hipólito, 2004). Grandes corporações estão entre as principais representantes deste grupo. Por último, as organizações com a cultura Dionísio, onde os talentos individuais têm espaço para florescer, as estratégias de remuneração devem dar ênfase na qualidade da remuneração, assim como, na competitividade da remuneração em relação ao mercado (Plothow, 2006).

Dessa forma, a competência essencial da organização será preservada e problemas não previstos no planejamento organizacional serão antecipadamente solucionados, tendo em vista que, conforme expressa Barton (2006), a estratégia de remuneração é complementar a cultura organizacional, onde os componentes de remuneração são culturalmente forjados. Dessa forma, desenvolver estratégias de remuneração ou dos demais subsistemas de Recursos Humanos, sem levar em consideração a cultura organizacional é criar um conflito cultural que dificulta a implementação de estratégias organizacionais. Assim, recomendamos aos gestores monitorar a cultura organizacional e identificar as suas características para desenhar as estratégias de Recursos Humanos, em particular, e as da organização em geral.

Finalizando, devem ser consideradas algumas limitações do estudo. Seria interessante estender esta pesquisa a uma amostra maior e com um maior número de respondentes para avaliar o construto da cultura organizacional. 
Giuseppe Maria Russo, Patricia Amelia Tomei, Antonio José Braga Linhares \& Andre Moreira Santos

\section{Agradecimentos}

Gostaríamos de agradecer ao Presidente do GRUPISA, Carlos Monnerat Rocha e ao Gerente de Operações, Carlos Amaury M. de Oliveira, pelo apoio e divulgação da pesquisa.

\section{REFERÊNCIAS}

ABBAD, G.; TORRES, C.V. Regressão múltipla stepwise e hierárquica em Psicologia Organizacional: aplicações, problemas e soluções. Estudos de Psicologia, 7 (número especial), 19-29, 2002.

ANKRAH, N.A.; LANGFORD, D.A. Architects and contractors: a comparative study of organizational cultures. Construction Management and Economics, 23, 595-607, 2005.

BALDACCHINO, G. A clash of human resource management cultures: a micro-state case study. The International Journal of Human Resource Management, 8(4), 506-518, 1997.

BALKIN, D.B.; GOMEZ-MEJIA, L.R. Toward a Contingency Theory of Compensation Strategy. Strategic Management Journal, 8(2), 169-182, 1987.

BARBOSA, L. Cultura e Empresas. Rio de Janeiro: Jorge Zahar, 2002.

BARROS, B.T. Gestão à Brasileira. São Paulo: Atlas, 2003. 
Correlacionando tipos de cultura organizacional com estratégias de remuneração utilizando a tipologia de Charles Handy

BARTON, G.M. Culture at Work. Scottsdale: WorldatWork Press, 2006.

BATEMAN, T.S.; SNELL, S.A. Administração: Construindo Vantagem Competitiva. São Paulo: Atlas, 1998.

BIRKINSHAW, J., HOOD, N.; JONSSON, S. Building firm-specific advantages in multinational corporations: The role of subsidiary initiative. Strategic Management Journal, 19(3), 221-242, 1998.

BOURANTAS, D.; ANAGNOSTELIS, J.; MANTES, Y.; KAFALAS, A.G. Culture Gap in Greek Management. Organization Studies, 11(2), 261-283, 1990.

BOURANTAS, D.; PAPADAKIS, V. Greek Management: diagnosis and prognosis. International Studies of Management and Organization, 26(3), 13-32, 1997.

CABLE D.M.; JUDGE, T.A. Person-organization Fit, Job Choice Decisions, and Organizational Entry. Organizational Behavior and Human Decision Processes, 67(3), 294-311, 1996.

CAMARA, P. Os Sistemas de Recompensas e a Gestão Estratégica de Recursos Humanos. Lisboa: Publicações Dom Quixote, 2000.

CAMERON, K.S.; QUINN, R.E. Diagnosing and Changing Organizational Culture: based on the competing values framework. New York: Addison-Wesley Publishing, 1999.

CLOSS, S.J. On the Factoring and Interpretation of Ipsative Data. Journal of Occupational and Organizational Psychology, 69, 41-47, 1996. 
Giuseppe Maria Russo, Patricia Amelia Tomei, Antonio José Braga Linhares \& Andre Moreira Santos

COHEN, J. Statistical Power Analysis for the Behavioral Sciences. Hillsdale: Erlbaum, 1988.

CONYON, M.J.; PECK, S.I.; SADLER, G.V. Compensation Consultants and Executive Pay: Evidence from the United States and the United Kingdom. Academy of Management Perspectives, 23(1), 43-55, 2009.

CROUCHER, R.; GOODERHAM, P.; PARRY, E. The Influences on Direct Communication in British and Danish Firms: Country, Strategic HRM or Unionization? European Journal of Industrial Relations, 12, 267-286, 2006.

DAFT. R.L. Organizações: teoria e projetos. São Paulo: Pioneira, 2000.

DELANEY, J.T.; HUSELID, M.A. The impact of human resource management practices on perceptions of organizational performance. Academy Management Journal, 39(4), 949-969, 1996.

DELERY, J.; DOTY, D. Modes of Theorizing in Strategic Human Resource Management: tests of universalistic, contingency, and configurational performance predictions. Academy Management Journal, 39(4), 802-835, 1996.

DENISON, D.R.; MISHRA, A.K. Toward a Theory of Organizational Culture and Effectiveness. Organization Science, 6(2), 204-223, 1995.

FEY, C.F.; DENISON, D.R. Organizational Culture and Effectiveness: Can American Theory Be Applied in Russia? Organization Science, 14(6), 686-706, 2003. 
Correlacionando tipos de cultura organizacional com estratégias de remuneração utilizando a tipologia de Charles Handy

FIGUEIREDO FILHO, D.B.; SILVA JÚNIOR, J.A. da. $\square \square$ Desvendando os Mistérios do Coeficiente de Correlação de Pearson (r). Revista Política Hoje, 18(1), 115-146, 2009.

FREITAS, M.E. de. Cultura Organizacional: formação, tipologias e impactos. São Paulo: Makron, 1991.

GAINEY, T.W.; KELLEY, D.E.; HILL, J.A. Telecommuting's Impact on Corporate Culture and Individual Workers: examining the effect of employee isolation. SAM Advanced Management Journal, 64(4), 4-10, 1999.

GERINGER, J. M.; HERBERT, L. Control and performance of international joint ventures. Journal of International Business Studies, 20(2), 235-254, 1989.

GOMEZ-MEJIA, L. Structure and Process Diversification, Compensation Strategy, and Firm Performance. Strategic Management Journal, 13(5), 381-397, 1992.

GOMEZ-MEJIA, L.R.; BALKIN, D.B. Compensation, Organizational Strategy, and Firm Performance. Cincinnati: Southwestern, 1992.

GOMIDE JUNIOR, S.; MARTINS, M.C. Os Deuses da Administração: construção e validação de quatro escalas para medida de cultura organizacional. Psicologia: Teoria e Pesquisa, 13(3), 311-316, 1997.

GRUPISA. GRUPISA: Grupo de Permuta de Informações Salariais. Disponível em: $<$ www.grupisa.com.br>. Acessado em: 09/09/2009.

HAIR JR., J.F.; ANDERSON, R.E.; TATHAM, R.L.; BLACK, W.C. Multivariate Data Analysis. New Jersey: Prentice Hall, 1998. 
Giuseppe Maria Russo, Patricia Amelia Tomei, Antonio José Braga Linhares \& Andre Moreira Santos

HANDY, C. The Gods of Management. London: Pan, 1980.

HARRISON, R. Understanding your Organization's Character. Harvard Business Review, May-June, 119-128, 1972.

HEGGESTAD, E.D.; MORRISON, M., REEVE, C.L.; MCCLOY, R.A. Forced-choice Assessments of Personality for Selection: evaluating issues of normative assessment and faking resistance. Journal of Applied Psychology, 91(1), 9-24, 2006.

HENEMAN, R.L.; FISHER, M.M.; DIXON, K.E. Reward and Organizational Systems Alignment: An Expert System. Compensation Benefits Review, 33(18), 18-29, 2001.

HIPÓLITO, J.A.M. Recompensas em empresas que atuam no Terceiro Setor: Análise a partir de seus pressupostos orientadores. 2004. Tese (Doutorado) - USP, São Paulo, 2004.

IGO, T.; SKITMORE, M. Diagnosing the Organizational Culture of an Australian Engineering Consultancy Using the Competing Values Framework. Construction Innovation, 6, 121-139, 2006.

LAWLER III, E. Pay and Organization Development. Reading: Addison-Wesley, 1981.

LAWLER III, E. Strategic Pay: aligning organizational strategies and pay systems. San Francisco: Jossey-Bass, 1990.

LEE, C.; BEAMISH, P.W. The characteristics of performance of Korean joint ventures in LDCs. Journal International Business Studies, 26, 637-654, 1995. 
Correlacionando tipos de cultura organizacional com estratégias de remuneração utilizando a tipologia de Charles Handy

MAJUMDAR, S.K. The Impact of Size and Age on Firm-Level Performance: Some Evidence from India. Journal Review of Industrial Organization, 12(2), 231-241, 1997.

MARX, R.; SOAREZ, J.P.R.F. A Influência da Orientação à Geração de Valor ao Acionista nas Práticas de Gestão de Pessoas no Ambiente da Produção. Gestão \& Produção, 15(2), 215-230, 2008.

MAXIMIANO, A.C.A. Teoria Geral da Administração: da Escola Científica à Competitividade na Economia Globalizada. 2a Edição. São Paulo: Atlas, 2000.

MCCLOY, R.A.; HEGGESTAD, E.D.; REEVE, C.L. A Silk Purse from the Sow's Ear: Retrieving Normative Information From Multidimensional Forced-Choice Items. Organizational Research Methods, 8(2), 222-248, 2005.

MILGROM, P.; ROBERTS, J. Economics, Organization and Management. New Jersey, Prentice Hall, 1992.

MILKOVICH, G.T.; NEWMAN, J.M. Compensation. $2^{\text {nd }}$ Ed. Texas: Business, 1987.

MOREIRA, L.F. Interconectando Estratégia, Recompensa e Performance. Anais do XV Congresso de Administração do Mercosul. Foz do Iguaçu, Brasil, 2008.

NAVES, E.M.R.; COLETA, M.F.D. Cultura e Comprometimento Organizacional em Empresas Hoteleiras. Revista de Administração Contemporânea, 7 (Edição Especial), 205$222,2003$. 
Giuseppe Maria Russo, Patricia Amelia Tomei, Antonio José Braga Linhares \& Andre Moreira Santos

O'NEAL, S. R ${ }^{2}$ : The Reward and Recognition Phenomenon. Compensation \& Benefits Review, 24(6), 48-52, 1992.

O'REILlY, C.; CHATMAN, J.; CALDWELL, D. People and Organizational Culture: a Profile Comparison Approach to Assessing Person-Organization Fit. Academy of Management Journal, 34(3), 487-516, 1991.

PLOTHOW, C.B. de A. Fatores Contingenciais à Estratégia de Remuneração da Força de Vendas. 2006. Dissertação (Mestrado) - USP (Departamento. de Administração), São Paulo, 2006.

REA, L.M.; PARKER, R.A. Designing and Conducting Survey Research: a comprehensive guide. $2^{\text {nd }}$ Ed., New York: Jossey-Bass, 1997.

RUSSO, G.M. Diagnóstico da Cultura Organizacional. Rio de Janeiro: Campus/Elsevier, 2010 .

SCHEIN, E.H. (1992). Organizational Culture and Leadership. $2^{\text {nd }}$ Ed., San Francisco: Jossey-Bass.

SCHIEHLL, E.; MORISSETTE, R. (2000). Motivation, Measurement and Rewards from a Performance Evaluation Perspective. Revista de Administração Contemporânea, 4(3), 724.

SHAW, J.; DELERY, J.; JENKINS, D.; GUPTA, N. (1998). An organizational level analysis of voluntary and involuntary turnover. Academy Management Journal, 41(5), 511-525. 
Correlacionando tipos de cultura organizacional com estratégias de remuneração utilizando a tipologia de Charles Handy

SILVA, M.M.P.S.A. (2008). As práticas de recompensas: consequencias na percepção de justiça e satisfação dos trabalhadores. 2008. Dissertação (Mestrado) - Instituto Superior de Ciências do Trabalho e da Empresa (Departamento de Economia), Lisboa.

SROUR, R.H. (1998). Poder, Cultura e Ética nas Organizações. Rio de Janeiro: Campus.

TOMEI, P.A.; RUSSO, G.M.; ANTONACCIO, C.F.B. (2008). Cultura Empreendedora: guia prático para seleção de empreendedores. Rio de Janeiro: OfficeBook.

TOSI JUNIOR, H.L.; GOMEZ-MEJIA, L.R. 1994. CEO Compensation Monitoring and Firm Performance. The Academy of Management Journal, 37(4), 1002-1016.

TROMPENAARS, F. (1994). Nas Ondas da Cultura: como entender a diversidade cultural nos negócios. São Paulo: Educator.

WELTER, G.M.R.; CAPITÃO, C.G. (2007). Medidas Ipsativas na Avaliação Psicológica. Avaliação Psicológica, 6(2), 157-165. 